\title{
A LITTLE HELP FROM MY FRIENDS
}

\author{
ANTHONY G. O'FARRELL
}

This article is based on a talk given at a one-day meeting in NUI, Maynooth on the Fourth of April, 2008, held to honour David Walsh and Richard Watson.

\section{INTRODUCTION}

This is a tribute to my dear colleagues and friends David Walsh and Richard Watson, who were here before me in Maynooth, and who laboured with me in the day and the heat. They cheerfully shouldered with me a teaching load that would, apparently, kill the academics of today. The teaching load required, in order to ensure that our students were adequately trained, continued to be a problem until the presidency of Míchael Ledwith, in the early 1990's. It was not easy to pursue research while giving 275 lectures a year, but they gave it their best. At distinct times, both helped me in my investigations. David was sound on complex analysis and hard analysis, so we joined forces to tackle some problems that required technical estimates for integral kernels that solve the $\bar{\partial}$-problem. Richard had a sound background in algebra, and he worked with me on problems that could be addressed using algebras of smooth functions. Richard became my Ph.D. student, after a while, and then, after graduating, continued to work with me for a few years. My period of active collaboration with David was in the early eighties, and with Richard the nineties. Recently, both have taken some interest in my reversibility project.

Most of the sources referred to in what follows will be found in the references cited in our joint papers, which are listed in the bibliography below.

\section{A Way to Think of Complex Analysis}

Holomorphic functions are the solutions to the $\bar{\partial}$ equation

$$
\bar{\partial} f=0,
$$

Date: November 20, 2018. 
where

$$
\bar{\partial} f=\frac{\partial f}{\partial \bar{z}} d \bar{z}, \quad \frac{\partial f}{\partial \bar{z}}=\frac{1}{2}\left\{\frac{\partial f}{\partial x}+i \frac{\partial f}{\partial y}\right\} .
$$

We shall refer to both $\bar{\partial}$ and $\frac{\partial}{\partial \bar{z}}$ as "the $\bar{\partial}$ operator" (pronounced $d$-bar operator), as convenient.

The $\bar{\partial}$ operator is skew:

$$
\int_{\mathbb{C}} \phi \frac{\partial \psi}{\partial \bar{z}} d x d y=-\int_{\mathbb{C}} \psi \frac{\partial \phi}{\partial \bar{z}} d x d y
$$

whenever $\phi$ and $\psi$ belong to the space $\mathcal{D}$ of $C^{\infty}$ complex-valued functions on $\mathbb{C}$, having compact support.

The adjoint operator acts on distributions:

$$
\left\langle\phi,\left(\frac{\partial}{\partial \bar{z}}\right)^{*} f\right\rangle=\left\langle\frac{\partial}{\partial \bar{z}} \phi, f\right\rangle,
$$

whenever $\phi \in \mathcal{D}$, and $f$ belongs to $\mathcal{D}^{\prime}$. In view of the skewness, we define

$$
\frac{\partial f}{\partial \bar{z}}=-\left(\frac{\partial}{\partial \bar{z}}\right)^{*} f, \forall f \in \mathcal{D}^{\prime}
$$

so that the operator $\frac{\partial}{\partial \bar{z}}$ on $\mathcal{D}^{\prime}$ is the weak-star continuous extension of $\frac{\partial}{\partial \bar{z}}$ on $\mathcal{D}$, when we regard $\mathcal{D}$ as a subset of $\mathcal{D}^{\prime}$, under the identification of each $f \in L_{\text {loc }}^{1}(d x d y)$ with the distribution represented by $f$, given by

$$
\langle\phi, f\rangle=\int_{\mathbb{C}} \phi f d x d y, \forall \phi \in \mathcal{D} .
$$

Complex Radon measures (Borel-regular complex-valued measures on $\mathbb{C}$, having finite total variation on each compact subset of $\mathbb{C}$ ) also represent distributions. The measure $\mu$ acts continuously on the space $C_{\mathrm{cs}}^{0}$ of continuous complex-valued functions on $\mathbb{C}$ having compact support, and equipped with the usual inductive limit topology, via

$$
\langle\phi, \mu\rangle=\int_{\mathbb{C}} \phi f d \mu, \forall \phi \in C_{\mathrm{cs}}^{0}
$$

and hence restricts to a continuous linear functional on $\mathcal{D}$. If we identify $f \in L_{\text {loc }}^{1}$ with the measure $f d x d y$, then this generalises the previous remark. A measure is uniquely-determined by the corresponding distribution, because $\mathcal{D}$ is dense in $C_{\mathrm{cs}}^{0}$, so we may identify the measure and distribution, without fear of confusion.

The $\bar{\partial}$ operator is linear and translation-invariant. It is also elliptic: this means that it is almost invertible; more precisely it has finitedimensional kernel and cokernel, when restricted to a suitable space. When restricted to $\mathcal{D}^{\prime}$, it has a very big kernel, the space of all entire 
functions. This statement is a case of Weyl's Lemma: a distributional solution $u \in \mathcal{D}^{\prime}(U)$ (where $\mathcal{D}(U)$ is the space of $C^{\infty}$ complex-valued functions on $U$ ) of $\bar{\partial} u=0$ on an open set $U \subset \mathbb{C}$ is representable by a holomorphic function on $U$. This is a good thing, because it gives complex analysts a nontrivial field of study. But when restricted to $\mathcal{E}^{\prime}$, the dual of the space of $C^{\infty}$ functions on $\mathbb{C}$ with compact support, $\bar{\partial}$ is injective. The fundamental solution is the locally-integrable function $-1 / \pi z$, i.e.

$$
\frac{\partial}{\partial \bar{z}}\left(-\frac{1}{\pi z}\right)=\delta_{0}
$$

the point mass at 0 . For $\phi \in \mathcal{D}$, we have

$$
\frac{\partial}{\partial \bar{z}} \hat{\phi}=\phi
$$

where

$$
\hat{\phi}=\left(-\frac{1}{\pi z}\right) * \phi
$$

the Cauchy transform of $\phi$. We extend the transform to a map $\mathcal{E}^{\prime} \rightarrow \mathcal{D}^{\prime}$ by setting

$$
\langle\phi, \hat{f}\rangle=-\langle\hat{\phi}, f\rangle, \forall \phi \in \mathcal{D}, \forall f \in \mathcal{E}^{\prime} .
$$

We have

$$
\frac{\partial}{\partial \bar{z}} \hat{f}=f, \forall f \in \mathcal{E}^{\prime}
$$

In particular, for each $f \in \mathcal{E}^{\prime}$, the distribution $\hat{f}$ is (represented by) a holomorphic function off spt $f$, (the support of $f$. Because of all this, the Cauchy transform is intimately connected with analytic function theory, and one can use it to establish many interesting results.

\section{Some Holomorphic Approximation Theorems}

For $X \subset \mathbb{C}^{n}$, let $\mathcal{O}(X)$ denote the space of functions holomorphic near $X$. For compact Hausdorff $X$, let $C^{0}(X)$ denote the Banach space of all continuous, complex-valued functions on $X$, with the sup norm.

Theorem 3.1 (Hartogs-Rosenthal, 1931). Suppose $X \subset \mathbb{C}$ is compact and has area zero. Then $\mathcal{O}(X)$ is dense in $C^{0}(X)$.

Proof. By the Separation Theorem for Banach spaces, it suffices to show that

$$
L \in C^{0}(X)^{*} \cap \mathcal{O}(X)^{\perp} \Rightarrow L=0 .
$$

By the Riesz Representation Theorem, the dual $C^{0}(X)^{*}=M(X)$, the space of (complex, Radon) measures supported on $X$.

Fix $\mu \in M(X)$ with $\mu \perp \mathcal{O}(X)$, i.e. $\int f d \mu=0$ whenever $f \in \mathcal{O}(X)$. 
Regarding $\mu$ as a distribution on $\mathbb{C}$, we find that $\hat{\mu}$ is the locallyintegrable function given by

$$
\hat{\mu}(\zeta)=\frac{1}{\pi} \int \frac{d \mu(z)}{z-\zeta}, \forall \zeta \in \mathbb{C}
$$

Since the function $z \mapsto 1 /(z-\zeta)$ belongs to $\mathcal{O}(X)$ for $\zeta \notin X$, we have $\hat{\mu}=0 d x d y$-a.e., hence $\hat{\mu}=0$ as a distribution, hence

$$
\mu=\frac{\partial \hat{\mu}}{\partial \bar{z}}=0
$$

Corollary 3.2 (A. Browder). Suppose $X \subset \mathbb{C}^{n}$ is compact, and each coordinate projection of $X$ has area zero. Then $\mathcal{O}(X)$ is dense in $C^{0}(X)$.

Proof. Denote $z=\left(z_{1}, \ldots, z_{n}\right)$, and $z_{j}=x_{j}+i y_{j}$. Fix $j \in\{1, \ldots, n\}$. Let $\pi_{j}: z \mapsto z_{j}$. Then $\pi_{j}(X)$ has area zero, so by Hartogs-Rosenthal $z \mapsto x_{j}$ and $z \mapsto y_{j}$ are uniform limits of functions (depending only on $\left.\pi_{j}(z)\right)$ that are holomorphic on a neighbourhood of $\pi_{j}^{-1}\left(\pi_{j}(X)\right)$, and hence belong to $\mathcal{O}(X)$. Thus the uniform closure $A$ on $X$ of the algebra $\mathcal{O}(X)$ contains all the coordinate functions $x_{j}$ and $y_{j}$, so by La Valleé Poussin's extension of Weierstrass' Polynomial Approximation Theorem (a special case of the Stone-Weierstrass Theorem), we conclude that $A=C^{0}(X)$.

Consider the function spaces, for $0<\alpha<1$ and compact $X \subset \mathbb{C}^{n}$ :

$$
\operatorname{Lip}(\alpha, X)=\left\{f \in C^{0}(X): \sup _{z \neq w} \frac{|f(z)-f(w)|}{|z-w|^{\alpha}}<+\infty\right\},
$$

and

$$
\operatorname{lip}(\alpha, X)=\left\{f \in \operatorname{Lip}(\alpha, X): \sup _{0<|z-w|<\delta} \frac{|f(z)-f(w)|}{|z-w|^{\alpha}} \rightarrow 0 \text { as } \delta \downarrow 0\right\} .
$$

With a suitable norm, $\operatorname{Lip}(\alpha, X)$ becomes a Banach algebra, and the subspace $\operatorname{lip}(\alpha, X)$ is a closed subalgebra, equal to the closure of $\mathcal{D}$ in $\operatorname{Lip}(\alpha, X)$. The elements of the dual $\operatorname{lip}(\alpha, X)^{*}$ may be represented in a manner somewhat similar to the Riesz representation, as follows.

Fix any $a_{0} \in X$.

Given $L \in \operatorname{lip}(\alpha, X)^{*}$, there exist $\lambda \in \mathbb{C}$ and a measure $\mu$ on the product $X \times X$ having no mass on the diagonal, such that

$$
L f=\lambda f\left(a_{0}\right)+\int_{X \times X} \frac{f(z)-f(w)}{|z-w|^{\alpha}} d \mu(z, w),
$$


whenever $f \in \operatorname{lip}(\alpha, X)$. If $L 1=0$, then $\lambda=0$. For such $\lambda$, this permits us to represent $\hat{L}$ by integration against an $L_{\text {loc }}^{1}$ function:

$$
\hat{L}(\zeta)=\frac{1}{\pi} \int \frac{(w-z) d \mu(z, w)}{(\zeta-z)(\zeta-w)|z-w|^{\alpha}} .
$$

Using this, and essentially the same proof as given for the HartogsRosenthal Theorem, one obtains [6, p. 387]:

Theorem 3.3. If $X \subset \mathbb{C}$ is compact with area zero, then $\mathcal{O}(X)$ is dense in $\operatorname{lip}(\alpha, X)$ for $0<\alpha<1$.

Corollary 3.4. If $X \subset \mathbb{C}^{n}$ has all its coordinate projections of area zero, then $\mathcal{O}(X)$ is dense in $\operatorname{lip}(\alpha, X)$.

\section{Higher-Dimensional Cauchy Transforms}

The utility of the Cauchy transform in one dimension prompted people to seek a similar tool for problems of several complex variables. Here one must use forms. The kernel $-1 / \pi z$ must be replaced by a $(2 n-1)$-form of type $(n, n-1)$ :

$$
\Omega=\sum_{j=1}^{n} K_{j}(\zeta, z) d \bar{\zeta}_{1} \wedge \cdots d \bar{\zeta}_{j-1} \wedge d \bar{\zeta}_{j+1} \wedge \cdots \wedge d \bar{\zeta}_{n} \wedge d \zeta_{1} \wedge \cdots \wedge d \zeta_{n}
$$

such that

$$
\phi(z)=\int \Omega(\zeta, z) \wedge \bar{\partial} \phi(\zeta)
$$

holds for test functions $\phi$. A form $\Omega$ that does this is called a CauchyLeray-Fantappié form. There are many such forms, and depending on the end in view, one prefers one or another. There are also more complex forms, involving boundary terms (analogous to Pompeiu's formula), useful for specific purposes.

In joint work with David Walsh, and the late Ken Preskenis, we obtained the following [8]:

Theorem 4.1. Let $X \subset \mathbb{C}^{n}$ be compact and holomorphically-convex. Let $E \subset X$ be closed, and suppose that each point $a \in X \sim E$ has a neighbourhood $N \subset \mathbb{C}^{n}$ such that $X \cap N$ is a subset of a $C^{1}$ submanifold without complex tangents. Then

$$
\operatorname{clos}_{C^{0}(X)} \mathcal{O}(X)=C^{0}(X) \cap \operatorname{clos}_{C^{0}(E)} \mathcal{O}(X),
$$

and

$$
\operatorname{clos}_{\operatorname{Lip}(\alpha, X)} \mathcal{O}(X)=\operatorname{lip}(\alpha, X) \cap \operatorname{clos}_{\operatorname{Lip}(\alpha, E)} \mathcal{O}(X), \text { for } 0<\alpha<1 .
$$


In other words, approximation problems on $X$ reduce to approximation problems on the singular set $E \subset X$.

This generalised and extended to $\operatorname{Lip}(\alpha)$ earlier work of Range and Siu $(E=\emptyset)$, Weinstock ( $X$ polynomially-convex), and ourselves [7] (See below).

The proof comes down to showing that if a distribution $L$ that acts continuously on $\operatorname{lip}(\alpha, X)^{*}$ annihilates $\mathcal{O}(X)$, then $L$ is supported on $E$. To do this, one constructs a kernel $\Omega(\zeta, z)$ such that Equation (11) holds for $z$ on a neighbourhood $U$ of $X$ and $\phi \in \mathcal{D}(U)$, and a second kernel $\tilde{\Omega}(\zeta, z)$ such that $\tilde{\Omega}(\zeta, z)=\Omega(\zeta, z)$ for $z \in X$ and $\zeta \in U$, and $\tilde{\Omega}$ has coefficients $\tilde{K}_{j}(\zeta, z)$ that are holomorphic in $z \in U$ for each $\zeta \in U \sim X$, and have another technical property. This construction is based on work of Berndtsson, building on the special Bochner-Martinelli kernel. Then, representing $L$ as before by a measure $\mu$ on $X \times X$ with no mass on the diagonal, we can represent

$$
\begin{aligned}
& \langle\phi, \quad L\rangle \\
& =\int_{X \times X} \frac{1}{|z-w|^{\alpha}} \int_{U}\{\Omega(\zeta, z)-\Omega(\zeta, w)\} \wedge \bar{\partial} \phi(\zeta) d \mu(z, w) \\
& =\int_{U} \int_{X \times X} \frac{1}{|z-w|^{\alpha}}\{\Omega(\zeta, z)-\Omega(\zeta, w)\} d \mu(z, w) \wedge \bar{\partial} \phi(\zeta),
\end{aligned}
$$

by Fubini's Theorem. (There are substantial technical estimates involved in justifying this.)

It remains to show that

$$
\int_{X \times X} \frac{1}{|z-w|^{\alpha}}\{\Omega(\zeta, z)-\Omega(\zeta, w)\} d \mu(z, w)=0
$$

for almost all $\zeta \in U$. The fact that $\Omega(\zeta, z)=\tilde{\Omega}(\zeta, z)$ for $z \in X$ and that the latter is holomorphic in $z$, and the technical properties (the most important of which is an "omitted sector property") allow us to approximate each coefficient in the integral by elements of $\mathcal{O}(X)$, and gives the desired result. For the details, see [8].

Corollary 4.2 (Range-Siu). If $E=\emptyset$, then $\mathcal{O}(X)$ is dense in $C^{0}(X)$.

Corollary 4.3. Let $F \subset Y$, where $Y$ is a compact subset of $\mathbb{C}^{n}$ and $F$ is a closed subset of $Y$. Let $f$ be a $\mathbb{C}^{n}$-valued function defined on a neighbourhood of $Y$, let $X=f(Y)$ and $E=f(F)$. Suppose that $X$ is polynomially-convex, and the matrix $f_{\bar{z}}$ (with columns $\frac{\partial f}{\partial \bar{z}_{j}}$ ) is invertible on $Y \sim F$. Then

$$
\operatorname{clos}_{C^{0}(X)} \mathbb{C}[z, w]=C^{0}(X) \cap \operatorname{clos}_{C^{0}(E)} \mathbb{C}[z, w],
$$


and

$$
\operatorname{clos}_{\operatorname{Lip}(\alpha, X)} \mathbb{C}[z, w]=\operatorname{lip}(\alpha, X) \cap \operatorname{clos}_{\operatorname{Lip}(\alpha, E)} \mathbb{C}[z, w] .
$$

Equation 2 is due to Weinstock.

Corollary 4.4. Suppose $\rho$ is a $C^{2}$ strictly plurisubharmonic function on a neighbourhood of bdy $X$, where $X$ is a compact subset of $\mathbb{C}^{n}$, with interior $D$, and that bdy $X=\{z: \rho(z)=0\},\{z: \rho(z)<0\} \subset D$, and $E=\operatorname{clos} D$. Then Equations Q and 3 hold.

In this case, Equation 2 is due to Henkin and Leiterer.

\section{Extending Smooth Functions}

According to one view, Geometry is an aspect of Group Theory. But more accurately, Geometry is Ring Theory. To be absolutely precise, Geometry is Topological Ring Theory.

Let $M$ be a $C^{k}$ manifold, and $X \subset M$. Given $f: X \rightarrow \mathbb{R}$, when does there exist a $C^{k}$ function $\tilde{f}: M \rightarrow \mathbb{R}$ such that the restriction $\tilde{f} \mid X=f$ ? This problem arises in many applications, and has been studied since the 1930's, with important work of Whitney and Glaeser. Richard Watson and I studied it in the early 1990's drawing on some ideas of mine that go back to the 1970's.

We deal now with real-valued functions, real vector spaces and algebras, and $i$ is just an index, or multi-index, and not $\sqrt{-1}$ any more.

Let $C^{k}(M)$ now denote the algebra (under pointwise operations) of $C^{k}$ real-valued functions on $M$. This is a Frechet algebra (a complete metric algebra) with the natural topology. For $S \subset C^{k}(M)$, let

$$
S^{\perp}=\left\{L \in C^{k}(M)^{*}: L f=0, \forall f \in S\right\},
$$

where $C^{k}(M)^{*}$ denotes the space of continuous linear functionals $L$ : $C^{k}(M) \rightarrow \mathbb{R}$. Note that $C^{k}(M)^{*}$ is a module over $C^{k}(M)$. For $X \subset M$, let

$$
X_{\perp}=\left\{f \in C^{k}(M): f(a)=0, \forall a \in X\right\} .
$$

Let $a_{\perp}=\{a\}_{\perp}$, when $a \in X$. Each $X_{\perp}$ is an ideal in $C^{k}(M)$. The ideal $\left(a_{\perp}\right)^{k+1}$ is generated by products of $k+1$ elements of $a_{\perp}$. Its annihilator $\left(\left(a_{\perp}\right)^{k+1}\right)^{\perp}$ consists of the so-called " $k$-th order point differential operators". In local coordinates $\left(x_{1}, \ldots, x_{d}\right)$, each $\partial \in\left(\left(a_{\perp}\right)^{k+1}\right)^{\perp}$ takes the form

$$
\partial f=\sum_{|i| \leq k} \alpha_{i} \frac{\partial f}{\partial x_{i}}(a), \forall f \in C^{k}(M),
$$

where $i=\left(i_{1}, \ldots, i_{d}\right) \in \mathbb{Z}_{+}^{d}$ denotes a multi=index, $|i|=\sum_{j} i_{j}$, and $\alpha_{i} \in \mathbb{R}$ are constants depending on $\partial$, but not on $f$. 
The $k$-th order tangent space to $M$ at $a$ is defined as

$$
\operatorname{Tan}^{k}(M, a)=C^{k}(M)^{*} \cap\left(\left(a_{\perp}\right)^{k+1}\right)^{\perp},
$$

and the $k$-th order tangent space to $X$ at $a$ is defined as

$$
\operatorname{Tan}^{k}(M, X, a)=\operatorname{Tan}^{k}(M, a) \cap\left(X_{\perp}\right)^{\perp},
$$

the set of $k$-th order point differential operators $\partial$ at $a$ such that $\partial f$ depends only on the values of $f$ on $X$. The disjoint unions

$$
\begin{aligned}
T^{k}(M) & =\dot{U}_{a \in M} \operatorname{Tan}^{k}(M, a), \\
\text { and } & \\
T^{k}(M, X) & =\dot{U}_{a \in M} \operatorname{Tan}^{k}(M, X, a)
\end{aligned}
$$

are called the $k$-th order tangent bundle of $M$, and the $k$-th order tangent sheaf of $X$, respectively. The stalks $\operatorname{Tan}^{k}(M, X, a)$ have the structure of finite-dimensional modules over a finite-dimensional real algebra, and provide numerical $C^{k}$ invariants for the pair $(M, X)$, since the tangent construction behaves functorially. A $C^{k}$ function $F: M \rightarrow M^{\prime}$ between $C^{k}$ manifolds induces an algebra homomorphism

$$
F^{\#}:\left\{\begin{aligned}
C^{k}\left(M^{\prime}\right) & \rightarrow C^{k}(M) \\
g & \mapsto g \circ F
\end{aligned}\right.
$$

and a $C^{k}$-module homomorphism

$$
F_{\#}=\left(F^{\#}\right)^{*}: C^{k}(M)^{*} \rightarrow C^{k}\left(M^{\prime}\right)^{*} .
$$

If $F$ maps $X$ into $X^{\prime}$, then $F_{\#}$ maps the stalk $\operatorname{Tan}^{k}(M, X, a)$ to the stalk $\operatorname{Tan}^{k}\left(M^{\prime}, X^{\prime}, f(a)\right)$, and so induces a map $F_{*}: T^{k}(M, X) \rightarrow$ $T^{k}\left(M^{\prime}, X^{\prime}\right)$. We established the following [9]:

Theorem 5.1. Let $X$ be a closed subset of a $C^{k}$ manifold $M, f: X \rightarrow$ $\mathbb{R}$ be continuous, and

$$
\pi:\left\{\begin{aligned}
M \times \mathbb{R} & \rightarrow M \\
(x, y) & \mapsto x
\end{aligned}\right.
$$

be the projection. Then $f$ has a $C^{k}$ extension to $M$ if and only if the map

$$
\pi_{*}: T^{k}(M \times \mathbb{R}, f) \rightarrow T^{k}(M, X)
$$

is bijective.

This result, and the $k$-th order tangent concept, are not particularly difficult, but are completely fundamental for the extension problem. They reduce the extension problem to the problem of deciding whether or not two integral dimensions (of $\operatorname{Tan}^{k}(M \times \mathbb{R}, f,(a, f(a))$ and $\left.\operatorname{Tan}^{k}(M, X, a)\right)$ agree at each point $a \in X$. We were gratified by the favourable reception of our paper, which included a congratulatory 
letter from Malgrange. It remained a problem to come up with a constructive procedure for deciding the question. Whitney himself dealt with this in dimension one, for all $k$. We provided a way to do it in 1993, in case $k=1$, for all dimensions. In recent years, C. Fefferman and co-workers have gone as far as can be done in providing a constructive procedure for general $k$. See the website [5] where this monumental corpus may be downloaded. Their work employs, inter alia the $k$-th order sheaf we introduced and our result. Fefferman was unaware of our work, having taken the concept and result from the 2003 Inventiones paper of Bierstone, Milman and Pawluckii [4]. I supplied a copy of our paper to Pawlucki in December 1997, at his request. These authors included our paper among their references, but did not attribute the concept to us. They referred to our paper only in order to make a gratuitously dismissive remark about it. I am at a loss to understand this behaviour. They called the tangent sheaf the Zariski paratangent bundle (although it is not in general a bundle), and subsequently a number of authors have referred to it as the Zariski paratangent bundle of Bierstone, Milman and Pawlucki. The bundle $T^{k}(M)$ was originally introduced by Pohl and Feldman, but the sheaf $T^{k}(M, X)$ appeared first in our paper.

I must also mention the work of Declan O'Keeffe [10], who proved the analogous result for $C^{k+\alpha}$ extensions $(k \in \mathbb{N}, 0<\alpha<1)$, and who also used $T^{k}$ to study algebraic curve singularities in $\mathbb{C}^{2}$.

\section{Approximating $C^{\infty}$ Functions}

In conclusion, here is a brief summary of the joint work with the late Graham Allan, Grayson Kakiko and Richard, on Segal's problem. The problem called for a characterization of the closed subalgebras of the algebra $C^{\infty}(M, \mathbb{R})$, for a smooth manifold $M$. The problem is local, so that we may take $M=\mathbb{R}^{d}$. There is not much loss in generality in considering subalgebras that are topologically-finitely-generated, i.e. those of the form

$$
A(\Psi)=\operatorname{clos}_{C^{\infty}\left(\mathbb{R}^{d}\right)} \mathbb{R}[\Psi]=\operatorname{clos}_{C^{\infty}\left(\mathbb{R}^{d}\right)}\left\{g \circ \Psi: g \in C^{\infty}\left(\mathbb{R}^{r}, \mathbb{R}\right),\right.
$$

where $\Psi=\left(\psi_{1}, \ldots, \psi_{r}\right) \in C^{\infty}\left(\mathbb{R}^{d}\right.$. In 1950, Nachbin conjectured a solution, analogous to Whitney's Spectral Theorem for closed ideals. Let $\mathbb{R}\left[\left[x_{1}, \ldots, x_{n}\right]\right]$ denote the algebra of formal power series in $n$ indeterminates, let

$$
T_{a}^{\prime}: C^{\infty}\left(\mathbb{R}^{d}, \mathbb{R}^{r}\right) \rightarrow \mathbb{R}\left[\left[x_{1}, \ldots, x_{d}\right]\right]^{r}
$$


denote the truncated Taylor series map, for each $a \in \mathbb{R}^{d}$, and let $T_{a} f=$ $f(a)+T_{a}^{\prime} f$ be the full Taylor series. (Note that $x_{j}$ would have to be replaced by $\left(x_{j}-a_{j}\right)$ in these series in applications of Taylor's Theorem.)

In case $\Psi \in C^{\infty}\left(\mathbb{R}^{d}, \mathbb{R}^{r}\right)$ is injective, Nachbin's conjecture comes down to

$$
A(\Psi)=\bigcap_{a \in \operatorname{crit} \Psi} T_{a}^{-1} \mathbb{R}\left[\left[T_{a}^{\prime} \Psi\right]\right]
$$

which may be stated in loose terms as: $f \in A(\Psi)$ if and only if $f$ has the "right kind" of Taylor series at each point.

Theorem 6.1 (Tougeron 1971). Suppose that for each compact $K \subset$ $\mathbb{R}^{d}$ there exists $\alpha>0$ and $\beta>0$ such that

$$
|\Psi(x)-\Psi(y)| \geq \alpha|x-y|^{\beta}, \forall x, y \in K .
$$

Then Equation (4) holds.

Corollary 6.2. If $\Psi$ is injective and real-analytic, then Equation (4) holds.

Our main result was this [1]:

Theorem 6.3. If $\Psi$ is injective and $d=1$, then Equation (4) holds.

The proof involves some hard analysis, to oversome the problems around the accumulation points of the critical set.

We say that $\Psi$ is flat at $a$ if $T_{a}^{\prime} \Psi=0$. We also proved the following useful result [2]

Theorem 6.4. If $\Psi$ is injective and $f \in C^{\infty}\left(\mathbb{R}^{d}\right)$ is flat at each critical point of $\Psi$, then $f \in A(\Psi)$.

Corollary 6.5. If $\Psi$ is injective and flat on crit $\Psi$, then Equation (4) holds.

Corollary 6.6. If $\Psi$ is injective and crit $\Psi$ is discrete, then Equation (4) holds.

In further work [3], we studied $A(\Psi)$ as a Frechet algebra, for injective $\Psi$. We established that it is always regular, and that membership of $A(\Psi)$ is a local property.

\section{Notes}

It is convenient to take this opportunity to correct the definition of proxy distance given on p.49 of our paper [2] in the proceedings of the meeting at Blaubeuren. This should read: 
Definition Let $E \subset \mathbb{R}^{d}$ be closed and $\kappa_{m} \geq 1(m=0,1,2, \ldots)$. A function $d_{E}: \mathbb{R}^{d} \rightarrow[0,+\infty)$ that is $C^{\infty}$ on $\mathbb{R}^{d} i \sim E$ is called a $\left\{\kappa_{m}\right\}$ proxy distance for $E$ if

$$
\frac{1}{\kappa_{0}} d_{E}(x) \leq \operatorname{dist}(x, E) \leq \kappa_{0} d_{E}(x), \forall x \in \mathbb{R}^{d}
$$

and

$$
\left|D^{m} d_{E}(x)\right| \leq \kappa_{m} \cdot \operatorname{dist}(x, E)^{1-m}, \forall x \in \mathbb{R}^{d}, \forall m \geq 1 .
$$

No other change to the paper is needed, and it all remains true.

(The reason for the change is that $d_{E}$ cannot be $C^{\infty}$ on the whole of $\mathbb{R}^{d}$ when $\emptyset \neq E \neq \mathbb{R}^{d}$; in the subsequent application $d_{E}$ is always composed with functions $\phi$ that vanish near 0 , so $\phi \circ d_{E}$ is $C^{\infty}$ on $\mathbb{R}^{d}$.)

I would also like to point out to readers of [8] that the interesting case of Example 3.2 is when $X \neq \operatorname{int} \operatorname{clos} X$. It is even interesting when $X$ has no interior.

\section{REFERENCES}

[1] G.R. Allan, G. Kakiko, A.G. O'Farrell and R.O. Watson. Finitely-generated algebras of smooth functions, in one dimension. J. Funct. Anal. 158 (1998) 45874.

[2] G.R. Allan, G. Kakiko, A.G. O'Farrell and R.O. Watson. Algebras of smooth functions. In M. Mathieu (ed.) Banach Algebras '97 (Blaubeuren). de Gruyter, Berlin. 1998. pp. 43-53.

[3] G.R. Allan, G. Kakiko, A.G. O'Farrell and R.O. Watson. Localness of $A(\Psi)$ algebras. Math. Proc. Roy. Ir. Acad. 101A (2001) 61-70.

[4] E. Bierstone, P.D. Milman and W. Pawlucki. Differentiable functions defined in closed sets. A problem of Whitney. Invent. Math. 151 (2003) 329-52.

[5] C. Fefferman. Selected Papers. http://www.math.princeton.edu/facultypapers/Fefferman/

[6] A.G. O'Farrell. Annihilators of rational modules. J. Functional Analysis 19 (1975) 373-89

[7] A.G. O'Farrell, K.J. Preskenis and D. Walsh. Polynomial approximation on graphs. Math. Ann. 266 (1983) 73-81.

[8] A.G. O'Farrell, K.J. Preskenis and D. Walsh. Holomorphic approximation in Lipschitz norms. Contemporary Mathematics 32 (1984) 187-94.

[9] A.G. O'Farrell and R.O. Watson. The tangent stars of a set, and extensions of smooth functions. J. Reine u. Angew. Math. 430 (1992) 109-37.

[10] D. O'Keeffe. The tangent stars of a set and extensions of functions in Lipschitz classes. Math. Proc. Roy. Ir. Acad. 97A (1997) 5-13.

Matematics Department, Nui, Maynooth, Co. Kildare, Ireland 